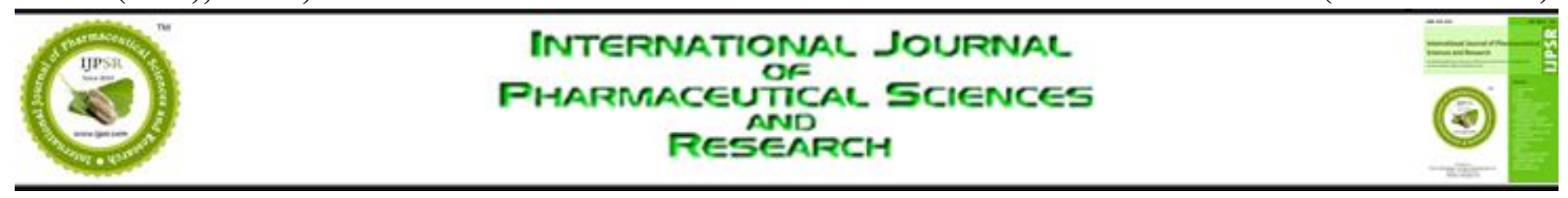

Received on 09 January, 2014; received in revised form, 08 March, 2014; accepted, 25 April, 2014; published 01 June, 2014

\title{
NANOROBOTS: A REVIEW
}

Preeti Khulbe

Department of Pharmaceutical Sciences, Bhimtal campus, Kumaun University, Nainital, Uttarakhand, India

Keywords:

Nanorobots, Targeted drug delivery, cancer therapy

\section{Correspondence to Author:}

Preeti Khulbe

Assistant Professor, Department of Pharmaceutical Sciences, Bhimtal campus, Kumaun University,

Nainital, Uttarakhand, India

E-mail: khulbe.preeti@yahoo.in
ABSTRACT: Nanomedicine offers the prospect of powerful new tools for the treatment of human diseases and the improvement of human biological systems. Nanomedicine is the process of diagnosing, treating, and preventing disease and traumatic injury, of relieving pain, and of preserving and improving human health, using molecular tools and molecular knowledge of the human body. Nanomedicine's nanorobots are so tiny that they can easily traverse the human body. Scientists report the exterior of a nanorobot will likely be constructed of carbon atoms in a diamondoid structure because of its inert properties and strength. Super-smooth surfaces will lessen the likelihood of triggering the body's immune system, allowing the nanorobots to go about their business unimpeded. Nanorobots can offer a number of advantages in drug delivery over present methods. These include more bioavailability, targeted therapy, fewer surgeon mistakes; reach remote areas in human anatomy, large interfacial area for mass transfer, non-invasive technique, this review focuses on the properties, method of preparations, mechanism of action, elements and applications of nanorobots. In addition, current study also involves the future aspects of nanorobots.
INTRODUCTION: The need for targeted drug delivery systems is increasing as today's biomedical technologies request new, innovative systems to replace difficult procedures.

By developing a micro-scale delivery system we hope to replace the need for traditional methods and instruments. Biomedical micro-robots are one possible solution to this and various other medical challenges.

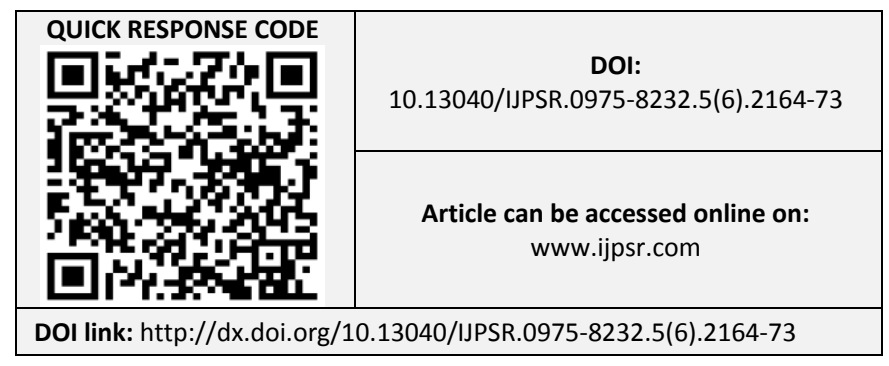

Nanomedicine offers the prospect of powerful new tools for the treatment of human diseases and the improvement of human biological systems by engineering nano/micro-scale robots that travel throughout the human body we can implement new technologies that re-define conventional processes 1 .

"Nanomedicine is the process of diagnosing, treating, and preventing disease and traumatic injury, of relieving pain, and of preserving and improving human health, using molecular tools and molecular knowledge of the human body.

Nanorobots would constitute any "smart" structure capable of actuation, sensing, signaling, information processing, intelligence, manipulation and swarm behavior at nano scale (10-9m). 
Bio nanorobots - Nanorobots designed (and inspired) by harnessing properties of biological materials (peptides, DNAs), their designs and functionalities ${ }^{2}$. These are inspired not only by nature but machines too. - Nanorobots could propose solutions at most of the nanomedicine problems.

Nanomedicine: Application of nanotechnology in medicine. Nanotechnology refers to the science and engineering activities at the level of atoms and molecules. A nanometer is a billionth of a meter, that is, about 1/80,000 of the diameter of a human hair, or 10 times the diameter of hydrogen atom. Nanorobots can offer a number of advantages over current methods such as;

(i) Use of nanorobot drug delivery systems with increased bioavailability;

(ii) Targeted therapy such as only malignant cells treated;
(iii)Fewer mistakes on account of computer control and automation;

(iv)Reach remote areas in human anatomy not operatable at the surgeon's operating table;

(v) As drug molecules are carried by nanorobots and released where needed the advantages of large interfacial area during mass transfer can be realized;

(vi)Non-invasive technique;

(vii) Computer controlled operation with nobs to fine tune the amount, frequency, time of release;

(viii) Better accuracy;

(ix)Drug inactive in areas where therapy not needed minimizing undesired side effects.

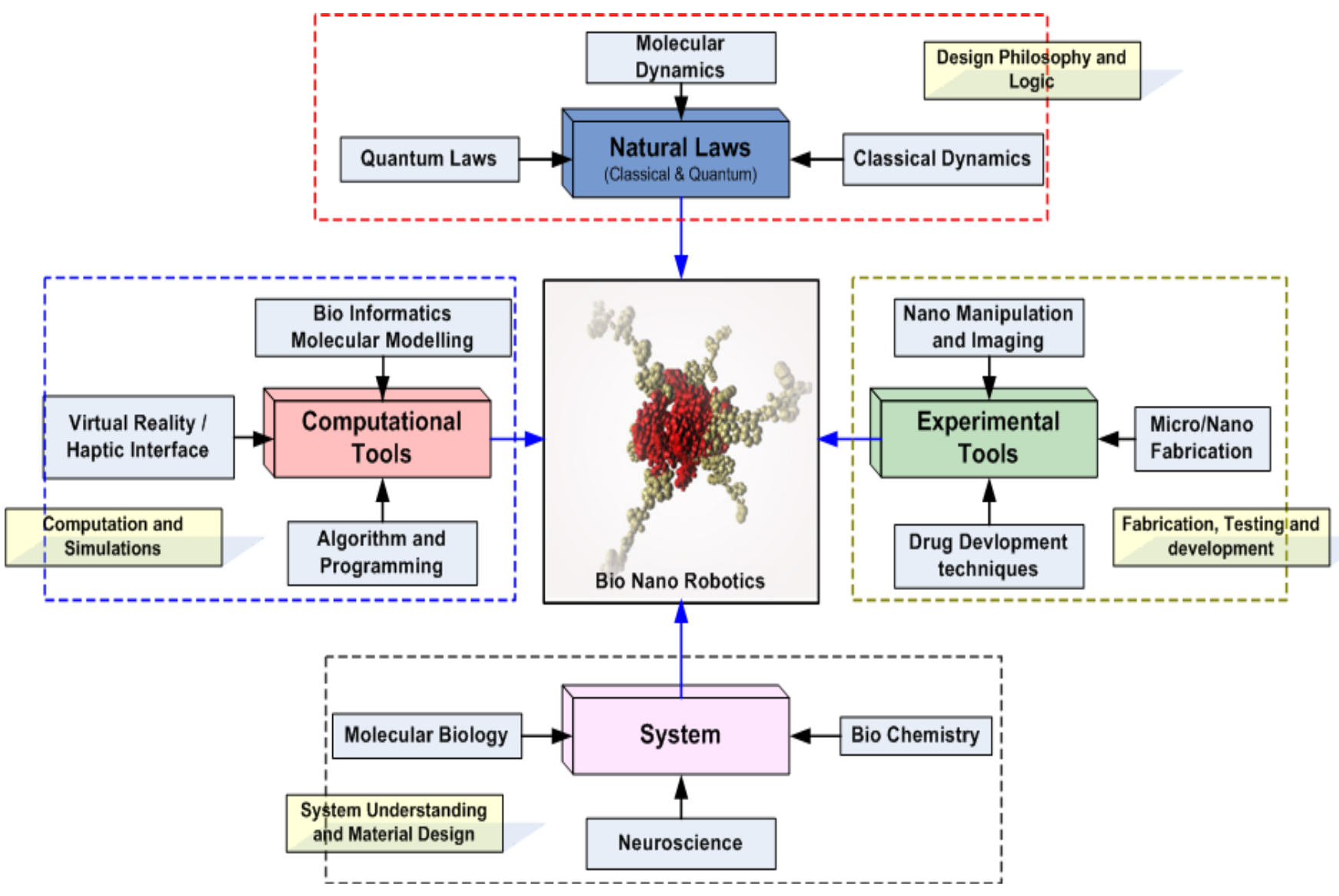

FIG. 1: NANO ROBOTICS; A TRULY MULTIDISCIPLINE FIELD ${ }^{6}$

The word "nanobot" (also "nanite", "nanogene", or "nanoant") is often used to indicate this fictional context and is an informal or even pejorative term to refer to the engineering concept of nanorobots.
The word nanorobot is the correct technical term in the nonfictional context of serious engineering studies. Some proponents of nanorobotics, in reaction to the grey goo scare scenarios that they 
earlier helped to propagate, hold the view that nanorobots capable of replication outside of a restricted factory environment do not form a necessary part of a purported productive nanotechnology, and that the process of self- replication, if it were ever to be developed, could be made inherently safe ${ }^{3}$. They further assert that free-foraging replicators are in fact absent from their current plans for developing and using molecular manufacturing.

TABLE 1: COMMERCIAL EFFORTS IN NANOMEDICINE

\begin{tabular}{cccccc}
\hline \multicolumn{7}{c}{ Product pipeline } & & & \\
\hline Healthcare sector & Number of products & Sales $\mathbf{~ \$ ~ b i l l i o n s ) ~}$ & Total & Advanced stages & Companies \\
\hline Drug delivery & 23 & 54 & 98 & 9 & 113 \\
Biomaterials & 9 & 0.07 & 9 & 6 & 32 \\
In vivo imaging & 3 & 0.02 & 8 & 2 & 13 \\
In vitro diagnostics & 2 & 0.78 & 30 & 4 & 35 \\
Active implants & 1 & 0.65 & 5 & 1 & 7 \\
Drug \& therapy & 0 & 0 & 7 & 1 & 7 \\
Total & 38 & 6.8 & 157 & 23 & 207 \\
\hline
\end{tabular}

Nanomedicine's nanorobots are so tiny that they can easily traverse the human body. Scientists report the exterior of a nanorobot will likely be constructed of carbon atoms in a diamondoid structure because of its inert properties and strength. Super-smooth surfaces will lessen the likelihood of triggering the body's immune system, allowing the nanorobots to go about their business unimpeded. Glucose or natural body sugars and oxygen might be a source for propulsion and the nanorobot will have other biochemical or molecular parts depending on its task ${ }^{4}$.

The first useful applications of nanomachines, if such are ever built, might be in medical technology, where they might be used to identify cancer cells and destroy them. Another potential application is the detection of toxic chemicals, and the measurement of their concentrations, in the environment. Recently, Rice University has demonstrated a single-molecule car which is developed by a chemical process and includes buckyballs for wheels ${ }^{5}$.

It is actuated by controlling the environmental temperature and by positioning a scanning tunneling microscope tip. Since nanorobots would be microscopic in size, it would probably be necessary for very large numbers of them to work together to perform microscopic and macroscopic tasks. These nanorobot swarms, both those which are incapable of replication (as in utility fog) and those which are capable of unconstrained replication in the natural environment (as in grey goo and its less common variants), are found in many science fiction stories, such as the Borg nanoprobes in Star Trek ${ }^{6}$.

\section{Disadvantages:}

- The initial design cost is very high.

- The design of the nanorobot is a very complicated one.

- Electrical systems can create stray fields which may activate bioelectric-based molecular recognition systems in biology.

- Electrical nanorobots are susceptible to electrical interference from external sources such as rf or electric fields, EMP pulses, and stray fields from other in vivo electrical devices.

- Hard to Interface, Customize and Design, Complex

- Nanorobots can cause a brutal risk in the field of terrorism. The terrorism and antigroups can make use of nanorobots as a new form of torturing the communities as nanotechnology also has the capability of destructing the human body at the molecular level.

- Privacy is the other potential risk involved with Nanorobots. As Nanorobots deals with the designing of compact and minute devices, there are chances for more ${ }^{1}$

Elements: Carbon will likely be the principal element comprising the bulk of a medical nanorobot, probably in the form of diamond or diamondoid/fullerene nanocomposites. 
Many other light elements such as hydrogen, sulfur, oxygen, nitrogen, fluorine, silicon, etc. will be used for special purposes in nanoscale gears and other components. The chemical inertness of diamond is proved by several experimental studies. Morphological examination revealed no physical damage to either fibroblasts or macrophages, and human osteoblast like cells confirming the biochemical indication that there was no toxicity and that no inflammatory reaction was elicited in vitro.

The smoother and more flawless the diamond surface, the lesser is the leukocyte activity and fibrinogen adsorption. The typical size of a blood born medical nanorobot will be 0.5-3 micrometers as it is the maximum size that can be permitted due to capillary passage requirement. These nanorobots would be fabricated in desktop nanofactories specialized for this purpose. The capacity to design, build, and deploy large numbers of medical nanorobots into the human body would, make possible the rapid elimination of disease and the effective and relatively painless recovery from physical trauma.

Medical nanorobots can be of great importance in easy and accurate correction of genetic defects, and help to ensure a greatly expanded health span ${ }^{7}$. More controversially, medical nanorobots might be used to enhance natural human capabilities. However, mechanical medical nanodevices would not be allowed to self-replicate inside the human body, nor would there be any need for selfreplication or repair inside the human body since these nanobugs are manufactured exclusively in carefully regulated nanofactories with outmost precision.

\section{APPROACHES:}

1. Biochip: The joint use of nanoelectronics, photolithography, and new biomaterials, can be considered as a possible way to enable the required manufacturing technology towards nanorobots for common medical applications, such as for surgical instrumentation, diagnosis and drug delivery. Indeed, this feasible approach towards manufacturing on nanotechnology is a practice currently in use from the electronics industry.
So, practical nanorobots should be integrated as nanoelectronics devices, which will allow tele-operation and advanced capabilities for medical instrumentation ${ }^{8}$.

2. Nubots: Nubot is an abbreviation for "nucleic acid robots." Nubots are synthetic robotics devices at the nanoscale. Representative nubots include the several DNA walkers reported by Ned Seeman's group at NYU, Niles Pierce's group at Caltech, John Reif's group at Duke University, Chengde Mao's group at Purdue, and Andrew Turberfield's group at the University of Oxford.

3. Positional nanoassembly: Nanofactory Collaboration[6], founded by Robert Freitas and Ralph Merkle in 2000, is a focused ongoing effort involving 23 researchers from 10 organizations and 4 countries that is developing a practical research agenda specifically aimed at developing positionallycontrolled diamond mechanosynthesis and a diamondoid nanofactory that would be capable of building diamondoid medical nanorobots.

4. Bacteria based: This approach proposes the use biological microorganisms, like Escherichia coli bacteria. Hence, the model uses a flagellum for propulsion purposes. The use of electromagnetic fields are normally applied to control the motion of this kind of biological integrated device, although his limited applications. ${ }^{9}$

5. Open Technology: A document with a proposal on nanobiotech development using open technology approaches has been addressed to the United Nations General Assembly ${ }^{7,8}$. According to the document sent to UN, in the same way Linux and Open Source has in recent years accelerated the development of computer systems, a similar approach should benefit the society at large and accelerate nanorobotics development.

The use of nanobiotechnology should be established as a human heritage for the coming generations, and developed as an open technology based on ethical practices for peaceful purposes. 
MAKING NANOROBOTS: Research and design of drug-carrying nanorobots is not new. Scientists have already created nanobot prototypes by using advanced molecular design software to create nanostructures that can store various molecular cargo.

Using a method known as 'DNA origami', pioneered in 2006 by scientist Paul Rothemund from Caltech University in the U.S., scientists have been able to manipulate DNA material into specific shapes and even program the 3-D DNA structures to carry out very basic robotic tasks, such as fusing to other cells and operating within other DNA material.

However, the DNA nanorobots created so far have faced challenges in movement, activation and targeting of drug release. Although DNA nanorobots have already been programmed to carry cargo and work in conjunction with other nanorobots, this new study is the first time that structural techniques have been exploited by advanced computing functions to securely deliver treatment to specific diseased cells ${ }^{10}$.

HOW IT WORKS: Instead of building a single complex molecule to identify multiple features of a cell surface, Dr. Stojanovic and his colleagues at Columbia used a different, and potentially easier, approach based on multiple simple molecules, which together form a robot (or automaton, as the authors prefer calling it).

To identify a cell possessing three specific surface proteins, Dr. Stojanovic first constructed three different components for molecular robots. Each component consisted of a piece of double-stranded DNA attached to an antibody specific to one of the surface proteins. When these components are added to a collection of cells, the antibody portions of the robot bind to their respective proteins (in the figure, CD45, CD3, and CD8) and work in concert. ${ }^{11}$

On cells where all three components are attached, a robot is functional and a fourth component (labeled 0 below) initiates a chain reaction among the DNA strands. Each component swaps a strand of DNA with another, until the end of the swap, when the last antibody obtains a strand of DNA that is fluorescently labeled.
At the end of the chain reaction- which takes less than 15 minutes in a sample of human blood-only cells with the three surface proteins are labeled with the fluorescent marker.

"We have demonstrated our concept with blood cells because their surface proteins are well known, but in principle our molecules could be deployed anywhere in the body," Dr. Stojanovic said. In addition, the system can be expanded to identify four, five, or even more surface proteins.

Now the researchers must show that their molecular robots work in a living animal; the next step will be experiments in mice ${ }^{12}$.

1. The nanorobot prototype: To create their new nanorobot, the team used cadnano, a DNA computing software, to help them design a folded, 3-D hexagonal DNA nanorobot that is able to carry molecular 'cargo' within its structure. The folded DNA device has two DNA-aptamer 'locks' - known as staples - that close around the cargo material to keep it secure until the destination cells are reached.

The nanorobot's molecular locks are programmed to respond to specific key combinations of proteins on the cell surface, so the cargo can only be delivered when the intended cell's receptors have the right combination. "We can finally integrate sensing and logical computing functions via complex, yet predictable, nanostructures - some of the first hybrids of structural DNA, antibodies, aptamers and metal atomic clusters - aimed at useful, very specific targeting of human cancers and T-cells," said Douglas ${ }^{13}$.

2. A nano 'smart box': By combining advanced structural design with the DNA origami method, which enables the cargo to be locked within the structure using a basic, one-lock method, the team has developed a drug-delivery vehicle that has not one lock mechanism, but two. "The nanorobot described by Douglas ... is a 'smart box' for other molecules - a box that opens if and only if it detects keys for locks placed on its lid," said Rothemund, who was not involved in the study. "This means that if it is keyed to proteins on the surface of cancer cells, for example, then it becomes a method of 
delivering drugs to cancer cells (and only cancer cells), potentially drastically reducing side effects."

Rothemund added that Douglas and his colleagues even managed to put two different locks on the lid of the box, so that it will open only when both keys are encountered. ${ }^{14}$ "Because many kinds of cells share the same 'keys' on their surface and it is only the combination of keys which identifies them, this capability may be the only way to recognise and deliver drugs to certain kinds of cells," he said. "No other approach that I know of promises to create such "programmable drug delivery" ${ }^{15}$.

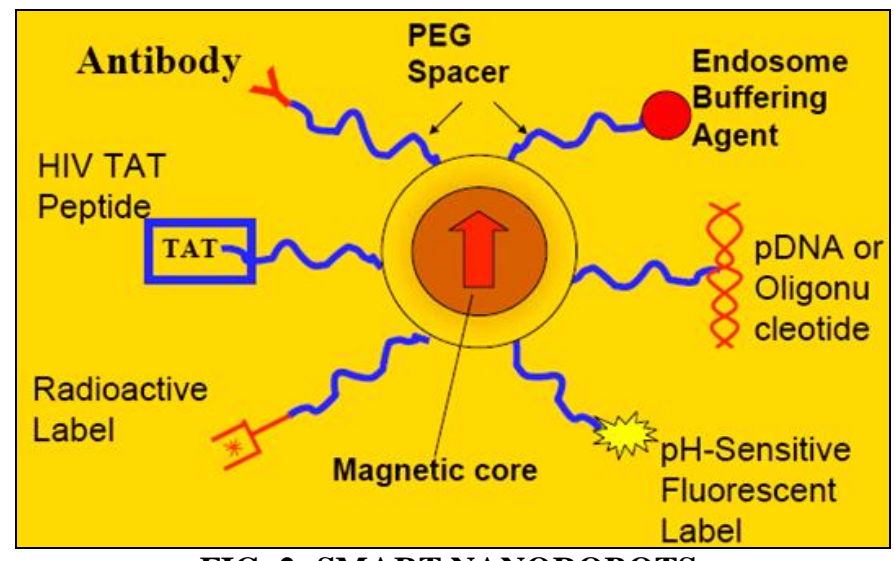

FIG. 2: SMART NANOROBOTS

\section{APPLICATION:-}

1. In Dentistry: The growing interest in the future of dental applications of nanotechnology is leading to the emergence of a new field called Nanodentistry. Nanorobots induce oral analgesia, Desensitize tooth; manipulate the tissue to re-align and straighten irregular set of teeth and to improve durability of teeth. Further it is explained that how nanorobots are used to do preventive, restorative, curative procedures.

Nanodental techniques involve many tissue engineering procedures for major tooth repair. Mainly nanorobotics manufacture and installation of a biologically autologous whole replacement tooth that includes both mineral and cellular components which leads to complete dentition replacement therapy ${ }^{16}$. Nanodentistry has given material that is nanostructured composite material, sapphire which increases tooth durability and appearance. Upper enamel layers are replaced by covalently bonded artificial material such as sapphire. This material has 100 to 200 times the hardness and failure strength than ceramic. Like enamel, sapphire is a somewhat susceptible to acid corrosion. Sapphire has best standard whitening sealant, cosmetic alternative. New restorative nano material to increase tooth durability is Nanocomposites. This is manufactured by nanoagglomerated discrete nanoparticles that are homogeneously distributed in resins or coatings to produce nanocomposites. The nanofiller include an aluminosilicate powder having a mean particle size of about $80 \mathrm{~nm}$ and a 1:4ratio of alumina to silica. The nanofiller has a refractive index of 1.503 , it has superior hardness, modulous of elasticity, translucency, esthetic appeal, excellent color density, high polish and 50\% reduction in filling shrinkage. They are superior to conventional composites and blend with a natural tooth structure much better ${ }^{17}$.

2. Nano Impression: Impression material is available with nanotechnology application. Nanofiller are integrated in the vinylpolysiloxanes, producing a unique addition siloxane impression material. The main advantage of material is it has better flow, improved hydrophilic properties hence fewer voids at margin and better model pouring, enhanced detail precision ${ }^{18}$.

3. Nanomedicine: Potential applications for nanorobotics in medicine include early diagnosis and targeted drug delivery for cancer biomedical instrumentation, surgery, pharmacokinetics, monitoring of diabetes, and health care ${ }^{9}$. In such plans, future medical nanotechnology is expected to employ nanorobots injected into the patient to perform treatment on a cellular level. Such nanorobots intended for use in medicine should be nonreplicating, as replication would needlessly increase device complexity, reduce reliability, and interfere with the medical mission ${ }^{19}$. Instead, medical nanorobots are posited to be manufactured in hypothetical, carefully controlled nanofactories in which nanoscale machines would be solidly integrated into a supposed desktop-scale machine that would build macroscopic products. 
4. The most detailed theoretical discussion of nanorobotics, including specific design issues such as sensing, power communication, navigation, manipulation, locomotion, and onboard computation, has been presented in the medical context of nanomedicine by Robert Freitas. Some of these discussions remain at the level of unbuildable generality and do not approach the level of detailed engineering ${ }^{20}$.

5. In Cancer Detection and Treatment: Cancer can be successfully treated with current stages of medical technologies and therapy tools. However, a decisive factor to determine the chances for a patient with cancer to survive is: how earlier it was diagnosed; what means, if possible, a cancer should be detected at least before the metastasis has begun ${ }^{21}$. Another important aspect to achieve a successful treatment for patients, is the development of efficient targeted drug delivery to decrease the side effects from chemotherapy. Considering the properties of nanorobots to navigate as bloodborne devices, they can help on such extremely important aspects of cancer therapy. Nanorobots with embedded chemical biosensors can be used to perform detection of tumor cells in early stages of development inside the patient's body ${ }^{22}$. Integrated nanosensors can be utilized for such a task in order to find intensity of E-cadherin signals.

Therefore a hardware architecture based on nanobioelectronics is described for the application of nanorobots for cancer therapy. ${ }^{23}$ Analyses and conclusions for the proposed model is obtained through real time $3 \mathrm{D}$ simulation.

6. In the diagnosis and treatment of Diabetes: Glucose carried through the blood stream is important to maintain the human metabolism working healthfully, and its correct level is a key issue in the diagnosis and treatment of diabetes. Intrinsically related to the glucose molecules, the protein hSGLT3 has an important influence in maintaining proper gastrointestinal cholinergic nerve and skeletal muscle function activities, regulating extracellular glucose concentration ${ }^{24}$. The hSGLT3 molecule can serve to define the glucose levels for diabetes patients.
The most interesting aspect of this protein is the fact that it serves as a sensor to identify glucose. The simulated nanorobot prototype model has embedded Complementary Metal Oxide semiconductor (CMOS) nanobioelectronics ${ }^{25}$.

It features a size of $\sim 2$ micronmeter, which permits it to operate freely inside the body. Whether the nanorobot is invisible or visible for the immune reactions, it has no interference for detecting glucose levels in blood stream. Even with the immune system reaction inside the body, the nanorobot is not attacked by the white blood cells due biocompatibility. For the glucose monitoring the nanorobot uses embedded chemosensor that involves the modulation of hSGLT3 protein glucosensor activity. Through its onboard chemical sensor, the nanorobot can thus effectively determine if the patient needs to inject insulin or take any further action, such as any medication clinically prescribed.

The image of the NCD simulator workspace shows the inside view of a venule blood vessel with grid texture, red blood cells (RBCs) and nanorobots. They flow with the RBCs through the bloodstream detecting the glucose levels ${ }^{26}$. At a typical glucose concentration, the nanorobots try to keep the glucose levels ranging around $130 \mathrm{mg} / \mathrm{dl}$ as a target for the Blood Glucose Levels (BGLs).

A variation of $30 \mathrm{mg} / \mathrm{dl}$ can be adopted as a displacement range, though this can be changed based on medical prescriptions. In the medical nanorobot architecture, the significant measured data can be then transferred automatically through the RF signals to the mobile phone carried by the patient. At any time, if the glucose achieves critical levels, the nanorobot emits an alarm through the mobile phone $^{27}$.

7. In Surgery: Surgical nanorobots could be introduced into the body through the vascular system or at the ends of catheters into various vessels and other cavities in the human body. A surgical nanorobot, programmed or guided by a human surgeon, could act as a semiautonomous on-site surgeon inside the human body ${ }^{28}$. 
Such a device could perform various functions such as searching for pathology and then diagnosing and correcting lesions by nanomanipulation, coordinated by an on-board computer while maintaining contact with the supervising surgeon via coded ultrasound signals ${ }^{29}$.

The earliest forms of cellular nanosurgery are already being explored today. For example, a rapidly vibrating $(100 \mathrm{~Hz})$ micropipette with a $<1$ micron tip diameter has been used to completely cut dendrites from single neurons without damaging cell viability. Axotomy of roundworm neurons was performed by femtosecond laser surgery, after which the axons functionally regenerated. A femtolaser acts like a pair of "nano-scissors" by vaporizing tissue locally while leaving adjacent tissue unharmed ${ }^{30}$.

8. Cryostasis: The extraordinary medical prospects ahead of us have renewed interest in a proposal made long ago: that the dying patient could be frozen, then stored at the temperature of liquid nitrogen for decades or even centuries until the necessary medical technology to restore health is developed. Called cryonics, this service is now available from several companies. Because final proof that this will work must wait until after we have developed a medical technology based on the foundation of a mature nanotechnology, the procedure is experimental. We cannot prove today that medical technology will (or will not) be able to reverse freezing injury 100 years from now. But given the wonderful advances that we see coming, it seems likely that we should be able to reverse freezing injury - especially when that injury is minimized by the rapid introduction through the vascular system of cryoprotectants and other chemicals to cushion the tissues against further injury ${ }^{31}$.

9. Diagnosis and Testing: Medical nanorobots can perform a vast array of diagnostic, testing and monitoring functions, both in tissues and in the bloodstream. These devices could continuously record and report all vital signs including temperature, pressure, chemical composition, and immune system activity, from all different parts of the body.
Nanorobots swallowed by a patient for diagnostic purposes approach the surface of the stomach lining to begin their search for signs of infection.

10. In Gene Therapy: Medical nanorobots can readily treat genetic diseases by comparing the molecular structures of both DNA and proteins found in the cell to known or desired reference structures. Any irregularities can then be corrected, or desired modifications can be edited in place. In some cases, chromosomal replacement therapy is more efficient than in cytorepair. Floating inside the nucleus of a human cell, an assembler-built repair vessel performs some genetic maintenance. Stretching a supercoil of DNA between its lower pair of robot arms, the nanomachine gently pulls the unwound strand through an opening in its prow for analysis. Upper arms, meanwhile, detach regulatory proteins from the chain and place them in an intake port ${ }^{32}$.

The molecular structures of both DNA and proteins are compared to information stored in the database of a larger nanocomputer positioned outside the nucleus and connected to the cell-repair ship by a communications link. Irregularities found in either structure are corrected and the proteins reattached to the DNA chain, which re-coils into its original form. With a diameter of only 50 nanometers, the repair vessel would be smaller than most bacteria and viruses, yet capable of therapies and cures well beyond the reach of present-day physicians. With trillions of these machines coursing through a patient's bloodstream, "internal medicine" would take on new significance. Disease would be attacked at the molecular level, and such maladies as cancer, viral infections and arteriosclerosis could be wiped out.

CONCLUSION: Nanorobots applied to medicine hold a wealth of promise from eradicating disease to reversing the aging process (wrinkles, loss of bone mass and age-related conditions are all treatable at the cellular level); nanorobots are also candidates for industrial applications. They will provide personalized treatments with improved efficacy and reduced side effects that are not available today. 
They will provide combined action - drugs marketed with diagnostics, imaging agents acting as drugs, surgery with instant diagnostic feedback 33. The advent of molecular nanotechnology will again expand enormously the effectiveness, comfort and speed of future medical treatments while at the same time significantly reducing their risk, cost, and invasiveness.

Nanorobots can offer a number of advantages in drug delivery over present methods. These include more bioavailability, targeted therapy, fewer surgeon mistakes; reach remote areas in human anatomy, large interfacial area for mass transfer, non-invasive technique, computer control of delivery, better accuracy, less side effects and greater speed of drug action.

Future healthcare will make use of sensitive new diagnostics for an improved personal risk assessment. Highest impact can be expected if those major diseases are addressed first, which impose the highest burden on the aging population: cardiovascular diseases, cancer, musculoskeletal conditions, neurodegenerative and psychiatric diseases, diabetes, and viral infections. Nanomedicine holds the promise to lead to an earlier diagnosis, better therapy and improved follow up care, making the health care more effective and affordable. Nanomedicine will also allow a more personalised treatment for many diseases, exploiting the in-depth understanding of diseases on a molecular level.

ACKNOWLEDGEMENT :- I acknowledge Mr. Vijay juyal and my parents for their support and motivation.

\section{REFERENCES:}

1. Hill, C., Amodeo, A ., Joseph, J.V. \& Patel, H.R.H; "Nano- and microrobotics: how far is the reality?". Expert Review of Anticancer Therapy 8(2008), (12): 1891-1897.

2. Couvreur, P. \& Vauthier, C. "Nanotechnology: Intelligent Design to Treat Complex Disease". Pharmaceutical Research 23(2006), (7): 1417-1450.

3. Cavalcanti, A. "Nanorobot Invention and Linux: The Open Technology Factor - An Open Letter to UNO General Secretary".(2009), CANNXS Project 1.

4. Vaughn JR. "Over the Horizon: Potential Impact of Emerging Trends in Information and Communication Technology on Disability Policy and Practice". National Council on Disability, Washington DC.: (2006),: 1-55.

5. Abhilash M; Nanorobots; International Journal of Pharma And Bio Sciences 2010, (1) : 105-115.

6. Renganathan Sharma ; Photodynamic Therapy of Alzheimer's Disease Using Intrathecal Nanorobot Drug
Delivery of Curcuma Longa for Enhanced Bioavailabilit; Journal of Scientific Research \& Reports 2(1): 206-227.

7. Tarakanov, A. O., Goncharova, L. B., Tarakanov Y. A. "Carbon nanotubes towards medicinal biochips". Wiley Interdisciplinary Reviews: Nanomedicine and Nanobiotechnology (2009). 2(1): 1-10.

8. Ignatyev, M. B. "N ecessary and sufficient conditions of nanorobot synthesis". Doklady Mathematics 82 (2010), (1): 671-675.

9. Scheufele, D. A., Lewenstein, B. V. "The Public and Nanotechnology: How Citizens Make Sense of Emerging Technologies". Journal of Nanoparticle Research 7 (2005). (6): 659-667.

10. Smith, D. M.; Goldstein, D. S.; Heideman, J. "Reverse Mergers and Nanotechnology".Nanotechnology Law \& Business (2007). 4 (3).

11. Cerofolini, G., Amato, P., Masserini, M., Mauri, G. "A Surveillance System for Early-Stage Diagnosis of Endogenous Diseases by Swarms of Nanobots". Advanced Science Letters 3(2010). (4): 345-352.

12. Yarin, A. L. "Nanofibers, nanofluidics, nanoparticles and nanobots for drug and protein delivery systems". Scientia Pharmaceutica Central European Symposium on Pharmaceutical Technology (2010). 78 (3): 542.

13. Wang, J. "Can Man-Made Nanomachines Compete with Nature Biomotors?". ACS Nano (2009). 3 (1): 4-9.

14. Amrute-Nayak, M., Diensthuber, R. P., Steffen, W., Kathmann, D., Hartmann, F. K., Fedorov, R., Urbanke, C., Manstein, D. J., Brenner, B., Tsiavaliaris, G. "Targeted Optimization of a Protein Nanomachine for Operation in Biohybrid Devices". Angewandte Chemie (2010). 122 (2): 322-326.

15. Patel, G. M., Patel, G. C., Patel, R. B., Patel, J. K., Patel, M. "Nanorobot: A versatile tool in nanomedicine". Journal of Drug Targeting (2010). 14 (2): 6367.

16. Wang, J. et al . "Micromachine Enables Capture and Isolation of Cancer Cells in Complex Media". Angew Chem. Int . Ed. 50: (2011). 4161-4165.

17. Richard P. Feynman (December 1959). "There's Plenty of Room at the Bottom". Retrieved March 2010.

18. . Wang, J. "Can Man-Made Nanomachines Compete with Nature Biomotors?". ACS Nano(2009). 3(1): 4-9.

19. Fisher, B. (2008). "Biological Research in the Evolution of Cancer Surgery: A Personal Perspective". Cancer Research 68 (24): 10007- 10020. 3. Cavalcanti, A., Shirinzadeh, B., Zhang, M. \& Kretly, L.C. "Nanorobot Hardware Architecture for Medical Defense". Sensors (2008). 8 (5):2932-2958.

20. . Elder, J.B., Hoh, D.J., Oh, B.C., Heller, A.C., Liu, C.Y. \& Apuzzo, M.L. "The future of cerebral surgery: a kaleidoscope of opportunities". Neurosurgery (2008). 62 (6): 1555-1579.

21. Martel, S., Mohammadi, M., Felfoul, O., Lu, Z. \& Pouponneau P. "Flagellated Magnetotactic Bacteria as Controlled MRItrackable Propulsion and Steering Systems for Medical Nanorobots Operating in the Human Microvasculature". International Journal of Robotics Research (2009). 28 (4): 571-582.

22. Sharma KR. Nanorobot Drug Delivery System for Cicumin for Treatment of Alzheimer's Disease with Increased Bioavailability during Treatment of Alzheimer's Disease, 68th Southwest Regional Meeting of the American Chemical Society, SWRMACS, Baton, Rouge, LA, Oct/Nov; 2012.

23. Sharma KR. Damped Wave Transport and Relaxation, Elsevier, Amsterdam, Netherlands; 2005.

24. Bird RB, Stewart WE, Lightfoot EN. Transport Phenomena, Revised Second Edition, John Wiley, Hoboken, NJ; 2007. 
25. Mickley HS, Sherwood TS, Reed CE. Applied Mathematical Methods in Chemical Engineering, McGraw Hill Professional, New York, NY; 1957. 227

26. Craig JJ. Introduction to Robotics: Mechanics and Control, Third Edition, Pearson Prentice Hall, Upper Saddle River, NJ; 2005.

27. Available: roboti/?lang=en\&output=json.

28. Sharma KR. Nanostructuing of Nanorobots for use in Nanomedicine, International Journal of Engineering \& Technology. 2012;2(2):116-134.

29. Available:http://ietjournals.org/archive/2012/feb_vol_2_no _2/6686111325866989.pdf.
30. Sharma KR. Nanostructuring Operations in Nanoscale Science and Engineering,McGraw Hill Professional; 2010, New York.

31. KlockeV. Nanrobot Module, Automation and Exchange, US Patent 2010/0140473 A1, 2010.

32. Regan BC, Zettl AK, Aloni S. Nanocrystal Powered Nanomotor, US Paten.t 2011, (7):863-798.

33. Dimitrov DV, Peng X, Xue SS, Wang D. Spin Oscillatory Device", US Patent 7,589, 600 B2, Seagate Technology, LLC; 2009.

34. W. Seifert LI. Samuelson BJ. Ohlsson and BorgstromLM. Directionally Controlled Growth of Nanowhiskers, US Patent. 2011, (7): 911-935.

\section{How to cite this article:}

Khulbe P: Nanorobots: A Review. Int J Pharm Sci Res 2014; 5(6): 2164-73.doi: 10.13040/IJPSR.0975-8232.5(6).2164-73

All @ 2013 are reserved by International Journal of Pharmaceutical Sciences and Research. This Journal licensed under a Creative Commons Attribution-NonCommercial-ShareAlike 3.0 Unported License.

This article can be downloaded to ANDROID OS based mobile. Scan QR Code using Code/Bar Scanner from your mobile. (Scanners are available on Google Playstore) 